\title{
Effects of Heart Rate Variability Biofeedback in Subjects with Stress-related Chronic Neck Pain: a Pilot study
}

\author{
David M. Hallman • Erik M.G. Olsson • Bo von Schéele • \\ Lennart Melin • Eugene Lyskov
}

D.M. Hallman

Centre for Musculoskeletal Research, University of Gävle, Sweden, Department of Public Health and Caring Sciences, Uppsala University

E-mail: david.hallman@hig.se

E.M.G. Olsson

Department of Public Health and Caring Sciences, Uppsala University

B. von Schéele

Biopsychosocial Medicine AB, Sweden

School of Innovation, Design and Engineering, Mälardalen University, Sweden

L. Melin

Department of Psychology, Uppsala University, Sweden

E. Lyskov

Centre for musculoskeletal research, University of Gävle, Sweden 
HRV Biofeedback in Neck Pain

\begin{abstract}
Recent studies focusing on autonomic nervous system (ANS) dysfunctions, together with theoretical pathophysiological models of musculoskeletal disorders, indicate the involvement of ANS regulation in development and maintenance of chronic muscle pain. Research has demonstrated the effectiveness of heart rate variability (HRV) biofeedback (BF) in increasing HRV and reducing the symptoms of different disorders characterized by ANS aberration. The study investigated the effects of resonance frequency HRV BF on autonomic regulation and perceived health, pain, stress and disability in 24 subjects with stress-related chronic neckshoulder pain. Twelve subjects participated in 10 weekly sessions of resonant HRV BF and were compared to a control group. Subjective reports and HRV measures during relaxation and in response to a standardized stress protocol were assessed for both groups pre- and postintervention. Group X time interactions revealed a significantly stronger increase over time in perceived health (SF-36) for the treatment group, including vitality, bodily pain and social functioning. Interactions were also seen for HRV during relaxation and reactivity to stress. The present pilot study indicates improvement in perceived health over a 10 week intervention with HRV-biofeedback in subjects with chronic neck-pain. Increased resting HRV as well as enhanced reactivity to hand grip and cold pressor tests might reflect beneficial effects on ANS regulation, and suggest that this intervention protocol is suitable for a larger controlled trial.
\end{abstract}

Keywords Heart rate variability $\cdot$ Biofeedback $\cdot$ ANS $\cdot$ Neck pain $\cdot$ Stress tests 
HRV Biofeedback in Neck Pain

\section{Introduction}

Neck and shoulder pain is one of the most frequently reported occupational complaints in Sweden (Statistics Sweden 2008) associated with psychosocial and mental stress (Bongers et al. 2002; Linton 2000; Lundberg 2002). The autonomic nervous system (ANS) is an important factor in pathogenesis of chronic muscle pain (Passatore \& Roatta 2006; Johansson et al. 2003; Maekawa et al. 2002). Elevated sympathetic activity may alter pain perception/sensitivity by increasing muscle tension (Roatta et al. 2008; Larsman et al. 2009) and impairing local microcirculation in the affected muscle (Larsson 1995). Furthermore, myofascial trigger points are characterized by increased spontaneous EMG activity, which may reflect sympathetic modulation of muscle spindle fibres (Hubbard \& Berkoff 1993; Gevirtz 2006). Recent studies have shown involvement of the ANS in localized chronic muscle pain. Generally, cardiovascular markers reflect increased sympathetic activity concomitant with vagal withdrawal at rest and altered reactivity to stress (Gockel et al. 1995; Kalezic et al. 2007, 2010; Leistad et al. 2008). However, there is a need for non-invasive behavioral treatment to improve ANS regulation and pain among those suffering.

Aspects of ANS function can be assessed using heart rate variability (HRV), which is also an acceptable biomarker of autonomic regulation (Task Force 1996). Antagonistic modulation of the heart via the parasympathetic and sympathetic branches of the ANS is reflected by fast and slow oscillations in beat-to-beat heart rate (Berntson et al. 1997). One way to increase HRV is through slow paced breathing using HRV biofeedback (BF) (Vaschillo et al. 2006). The amplitude of HRV is maximized during slow breathing, approximately six breaths per minute. The physiological mechanism involves baroreflex control of HR, compensating 
HRV Biofeedback in Neck Pain

for changes in blood pressure via the ANS. It has been suggested that resonance frequency breathing (i.e., the breathing frequency that causes the largest HR oscillation amplitude) may strengthen baroreflexes, thus improving the autonomic functioning (Vaschillo et al. 2002;

Lehrer et al. 2003). HRV BF has shown to be useful in treating symptoms in various disorders characterized by aberration of the ANS (Wheat and Larkin 2010), including posttraumatic stress disorder (PTSD) (Zucker et al. 2009), depression (Siepmann et al. 2008) and heart disease (Nolan et al. 2005) as well as musculoskeletal pain (Hasset et al. 2007; Kapitza et al. 2010). However, further controlled studies are needed on muscle pain. In order to investigate the possible effect of HRV BF on ANS regulation it is important to assess HRV during reactivity and recovery to various tests in addition to rest.

Therefore, the aim of the present pilot study was to investigate the effects of resonance frequency HRV BF on ANS regulation and perceived health, pain, stress and functional disability in subjects with stress-related chronic neck-shoulder pain. We hypothesize that 10 weeks of resonance HRV BF treatment in subjects with stress-related chronic neck pain will increase basal HRV and reduce symptoms, in comparison with a control group.

\section{Methods}

Subjects

The study included 24 subjects, 22 women and 2 men (mean age 40.5 years; range 25-50), reporting chronic neck and shoulder pain and perceived stress. Subjects were recruited 
HRV Biofeedback in Neck Pain

through the stress clinic (PBM Sweden), advertisements on the website, recommendations from associated physiotherapists and invitations to public service employees in two cities north of Stockholm, Sweden. Respondents underwent a structured telephone interview and filled in forms to ensure they met the case criteria for neck-shoulder pain and were then randomly assigned to an intervention or control group (see Table 1). From a total of 60 respondents (4 males, 56 females), 24 eligible subjects were included in the study. Inclusion criteria were: age between 20 and 50 years and perceived pain and/or other symptoms of muscle discomfort primarily located to the neck-shoulder area, observed for at least 6 months and persistently over the last six consecutive weeks. Diagnosis of neck-shoulder pain and stress related symptoms were evaluated by a specialized psychologist. Exclusion criteria were: regular use of medications known to affect ANS function or pain perception two weeks prior to participation including antidepressants, benzodiazepines, anti-inflammatory medications and betablockers. Subjects reporting diagnoses of rheumatism, diabetes, traumatic musculoskeletal system damage, chronic neurological and endocrinology syndromes as well as hypertension, coronary artery diseases, substance abuse and overweight (BMI>30) were also excluded. All subjects were informed of the study aim and the details of the clinical and experimental examinations. Before participation, subjects gave their written informed consent. The study was approved by the ethics committee at Uppsala University.

\section{Design and protocol}

The study employed a single-blind design. The experimenter performing the pre- and postintervention measurements did not know to which group (treatment vs. control) the subject 
HRV Biofeedback in Neck Pain

belonged. The treatment group participated in 10 weekly sessions of resonance HRV BF training led by a licensed psychologist. During Session 1 and 10, individual resonance frequency was assessed by having the subject breathe at various frequencies. The control group took part in the breathing protocol in Session 1 and 10, without any prescribed treatment in between. In order to evaluate treatment efficacy regarding subjective and physiological outcomes, both groups took part in an extensive assessment protocol the week before and after intervention.

\section{Resonance HRV biofeedback}

The purpose of the first training session was to asses each subject's resonance frequency. The procedure consisted of the subject breathing at various rates following a visual pacer, alternately set at $6.5,6,5.5,5$ and 4.5 breaths per minute for two minutes each. The paced breathing periods were separated by 2 minutes of free, non-paced breathing. HRV spectral power was computed and analyzed for each period, and additionally, the resonant frequency was defined as the respiratory frequency that produced the highest HRV in the low frequency band (LF HRV 0.04-0.15 Hz; Lehrer et al. 2000). The first session started with a 10-minute adaptation period during which the subject was comfortably seated in an armchair, hooked up to the equipment, and given an explanation of the test procedure. This procedure was later repeated at Session 10 for both groups.

For Session 2-9, the respiratory pacer was set at the particular frequency found in the previous session. Each session included four five-minute periods of resonant breathing with two mi- 
HRV Biofeedback in Neck Pain

nutes of rest after each period. In order to maximize HRV during the training sessions, small adjustments in individual breathing frequency were made, as previously described (Vaschillo et al. 2006). Subjects received visual HRV feedback during resonance frequency breathing. They were instructed to try to maximize their peak-to-peak HRV as well as to attain the phase between respiration and $\mathrm{HRV}$ changes as closely as possible. In each session, $\mathrm{ETCO}_{2}$ was assessed to ensure that the subject was not hyperventilating. If $\mathrm{ETCO}_{2}$ fell below $3.5 \%$, the subject was reminded to breathe less deeply. Between sessions, subjects were instructed to practice paced breathing for at least 15 minutes a day, five days a week, at home. They were instructed on how to use a regular watch as a pacer and were also given pacer software to use on their home computer (EZ-air, downloadable from www.bfe.org). Controls were instructed to perform their usual activities and were not refrained from any pharmalogical or behavioural treatment, besides those stated as exclusion criteria.

\section{Stress tests}

Assessment of ANS regulation under the resting condition and reactivity to stress, together with self-estimates, was carried out the week before and directly after the 10-week resonant HRV biofeedback training. Specific functional tests were selected to target different aspects of autonomic reactivity. Baseline HRV was obtained after 10 minutes of rest, followed by a hand grip test (HGT), a cold pressor test (CPT) and a deep breathing test (DBT), with a 5minute recovery period after each test. The subjects were instructed to relax and breathe normally, i.e. not to practice slow breathing. Examinations were conducted in the morning between 8:00-10:00 or in the afternoon between 13:00-15:00, and each subject was assessed at 
HRV Biofeedback in Neck Pain

the same time of day on all occasions. During the continuous measurement the subjects were seated in a comfortable chair, adjusted for each person to a semi-reclined position. The temperature in the room was approximately $23^{\circ} \mathrm{C}$ and the light was dimmed to provide a relaxing atmosphere. Beforehand, subjects were asked to refrain from smoking, caffeine intake, eating and drinking two hours prior to examinations.

The hand grip test (HGT) was used as a physical stressor. During this test, the subjects performed a maximum voluntary contraction by pressing a dynamometer with their dominant hand and then maintaining their grip at a level of 30 percent of maximum voluntary force for three minutes or as long as possible. The HGT is a sensitive test of sympathetic function (Khurana \& Setty 1996).

The cold pressor test (CPT) induces a sympathetic response predominantly mediated by nociceptive C-fibres (Lovallo 1975). CPT was carried out by immersing the subjects' dominant hand in cold water, approximately $3^{\circ}$ Celsius, for a maximum of three minutes or as long as possible. The subjects avoided isometric contraction, hyperventilation or held expiration during the test. The CPT is a widely used and documented test of sympathetic function (Fagius et al. 1989; Victor et al. 1987).

The deep breathing test (DBT) causes large oscillations in heart rate, which is modulated by activity of the baroreflex (Davies et al. 2002). DBT was carried out by subjects breathing 6 breaths during one minute, following a paced stimulus presented visually on a computer screen and auditorily from two speakers (i.e., Breathe in - Breathe out). The difference be- 
HRV Biofeedback in Neck Pain

tween the largest R-R interval and the shortest R-R interval for each of the six breathing phases was calculated.

Stress reactivity was defined as the change in HRV over time (test-baseline) and recovery as the change from test to resting condition (rest-test). Frequency domain parameters of HRV were extracted from 2-minute segments. Two subjects in the treatment group failed to complete 2 minutes of the HGT, and only seven subjects in the treatment group and 8 controls fulfilled CPT. Therefore, 60 second segments were used when analyzing the time domain measures of HRV for these tests. The 60s segments were averaged over a 4-minute period during baseline.

Measures

Questionnaires

Health-related quality of life was assessed using the Short Form 36 Item Health Survey (SF36) whose proven reliability and validity extend to the Swedish translation (Ware et al. 1998). It contains 36 items and includes eight different subscales addressing different aspects of mental and physical health (i.e., physical function, physical role, bodily pain, general health, vitality, social function, emotional role and mental health). The subscales range from 0 to 100 , with higher score indicating better health. 
HRV Biofeedback in Neck Pain

Pain intensity was assessed using the Borg CR10 scale. Subjects were asked to report their perceived pain in the neck/shoulder area "currently", i.e. when they arrived for the laboratory examination, and "for the past six months" on separate days. The scale ranges from 0 "not at all" to 10 "almost unbearable", and shows satisfactory reliability and validity (Borg 1992).

Stress related symptoms were assessed using the stress medicine symptom scale (SMSS). Subjects rated the frequency and impact of 14 symptoms on a scale from 0 to 7 . A summation was used as an index of total symptom report, which ranged from 0 to 98 . SMSS has recently shown good reliability and acceptable validity.

Disability due to neck-shoulder pain was assessed using the neck disability index (NDI) (Vernon \& Mior 1991). NDI is a short questionnaire that contains 10 items on the extent to which pain is affecting the individual in different aspects of daily life (i.e., reading, driving lifting, sleeping, etc.). The scale ranges from 0-100, with higher scores indicating more disability. NDI is the most commonly used self-report measure for neck pain and good reliability and validity have been reported (McDermid et al. 2009).

Levels of anxiety and depression were measured using the Hospital Anxiety and Depression Scale (HAD). HAD consists of a depression and anxiety subscale. Scores on each subscale range from 0 to 21 . A score above 7 but below 11 is interpreted as mild anxiety or depression, and a score above 10 is interpreted as clinical anxiety or depression (Zigmond and Snaith, 1983). Good psychometric properties have been reported for this instrument that extends to the Swedish translation (Lissbergs, Nygren, and Soderman, 1997). 
HRV Biofeedback in Neck Pain

Physiological recordings

During the stress tests, a bipolar electrocardiogram was recorded using the Biopac system (Biopac Systems inc, USA) with electrodes placed on the sixth left rib and the distal end of sternum; the signal was sampled at $2000 \mathrm{~Hz}$, bandpass filtered $(0.05-200 \mathrm{~Hz})$ and amplified 500 times. After visual inspection and editing of ectopic beats, normal R-R intervals were further analyzed in both the time domain (i.e., inter-beat intervals (IBI); standard deviation of normal beat-to-beat intervals (SDNN); proportion of successive differences $>50 \mathrm{~ms}$ between IBIs (pNN50)) and the frequency domain (LF HRV, 0.05-0.15 Hz; HF HRV, 0.15-0.4 Hz, LF/HF ratio), according to Task Force standards (Task Force of the European Society of Cardiology and The American Society of Pacing and Electrophysiology, 1996). HRV, end tidal $\mathrm{CO}_{2}\left(\mathrm{ETCO}_{2}\right), \mathrm{SpO}_{2}$ at the finger, respiration rate, finger temperature, and skin conductance were measured continuously during HRV BF protocols using J\&J-Engineering I-330-C-2 Physiological Monitoring System (J\&J Engineering; Poulsbo, WA), an Air-Pas oxycapnograph (PBM, Stockholm, Sweden), and cStress customized software (PBM, Stockholm, Sweden).

Statistical analyses

T-tests and Chi square analyses were conducted in order to investigate similarity between the groups in patient characteristics pre-intervention. Repeated measures analyses of variance 
HRV Biofeedback in Neck Pain

ANOVA (group X time) were carried out to test treatment efficacy with regard to improving self reports. A significant interaction (group X time) was interpreted as an effect of treatment, while a time effect (pre - post) was interpreted as a regression towards the mean. Additional exploratory ANOVA (group X time) were conducted for HRV at rest and for the responses to HGT, CPT and DBT. All statistical analyses were carried out using SPSS software installed on a PC. The alpha level was $p \leq 0.05$.

\section{Results}

During baseline both groups showed comparable distributions of age, gender, BMI and pain (Table 1). One subject in the control group dropped out after the first session and one control subject did not return for the stress assessment after 10 weeks, but did fill in the questionnaires. All subjects in the treatment group completed the measurements before and after treatment.

Please, insert Table 1 about here

Questionnaires

SF-36 
HRV Biofeedback in Neck Pain

The SF-36 scores were similar between groups before the intervention started. For the SF-36 sub-indices, significant interaction effects (group X time) were seen for vitality $(p=0.005$ ), social function $(p=0.047)$ and bodily pain $(p=0.049)$ in increased ratings over time for the treatment group compared to controls. Ratings of physical function, bodily pain and vitality were affected by time, indicating an increase over time for both groups $(p<0.05)$ (Table 2$)$.

Stress-related symptoms

No group differences in SMSS were found before the intervention. The sum of total symptoms did not show significant time or interaction (group X time) effects (Table 2).

Pain and disability

Pain intensity in the neck-shoulder region, assessed using the Borg CR10 scale, decreased after 10 weeks, as revealed by an effect of time $(p=0.009)$. There was no interaction effect (group X time). NDI scores significantly decreased by time $(p=0.012)$ with no significant interaction effect (Table 2).

HAD

The level of depression and anxiety did not differ significantly between groups before the intervention. Ratings of perceived anxiety and depression were reduced after 10 weeks, as 
HRV Biofeedback in Neck Pain

revealed by main effects of time $(p<0.05)$. No effect of treatment (group X time) was seen (Table 2).

Please, insert Table 2 about here

Stress tests

Resting HRV

Results of resting HRV revealed no significant differences between groups in autonomic tone before the intervention. After the intervention, the treatment group tended to increase their resting HRV, whereas controls showed lower HRV. The interaction effect (group X time) was significant for LF power $(p=0.016)$ but no other measure of HRV reached significance. An ANOVA did not reveal any effects of time (Table 3).

Hand grip

To evaluate whether the treatment had any effect on sympathetic to parasympathetic regulation during reactivity and recovery from stress, additional ANOVAs were conducted ( roup X time) for all HRV variables. The amplitude of the HRV response to HGT increased with time for the treatment group as compared to the control group. ANOVA revealed significant inte- 
HRV Biofeedback in Neck Pain

ractions (group X time) for IBI $(p=0.011), \operatorname{pNN} 50(p=0.050)$ and LF power $(p=0.023)$ (Table 4). Effects of time were not seen. Recovery from stress showed an interaction effect (group X time) for SDNN ( $p=0.039$ ) but did not reach significant levels for IBI, LF, HF and LF/HF ratio. No main effects of time were seen.

Please, insert Figure 1 about here

Cold pressor

Interaction effects (group $\mathrm{X}$ time) for time domain measures of reactivity to $\mathrm{CPT}$ indicated that the treatment group increased their HRV response to CPT after 10 weeks of HRV BF compared to the control group: IBI $(p=0.02)$ and pNN50 $(p=0.005)$. The frequency domain measures of HRV were non-significant in the ANOVA, and the effects of time were not significant for any HRV measure (Table 4). Recovery from CPT did not reveal any interaction effects (group X time) or main effects time.

Please, insert Table 3-4 about here 
HRV Biofeedback in Neck Pain

Deep breathing

Mean HR peak-to-peak amplitude during DBT prior to treatment was similar in both groups (Treatment 20.1 Control 17.4 beats/min). Including the post-test in an ANOVA (Treatment 19.5 Control 16.3) did not reveal any significant effect of time or interaction (group X time) Resonance frequency $($ mean $=5.7 / \mathrm{min}$ ) was equal in both groups before treatment and did not change across sessions, indicating the stability of respiratory frequency during treatment.

\section{Discussion}

The present study investigated the effects of HRV resonance biofeedback on autonomic regulation and self-assessed health, disability, and pain in subjects with stress-related chronic neck-shoulder pain.

Resting heart rate variability

The present study found relatively small but significant treatment-related differences in HRV between groups. For resting HRV, only low frequency (LF) power increased significantly more in the intervention group after 10 weeks of HRV BF, in comparison with controls. LF has been suggested to reflect both PNS and SNS modulation of the heart, possibly mediated by baroreflex control (Berntson et al., 1997; Task Force 1996). Thus, we do not know if the results reflect reduced sympathetic or increased parasympathetic tone. Lehrer et al. (2003) 
HRV Biofeedback in Neck Pain

investigated the physiological effects of HRV BF in healthy adults and found a session-bysession increase in LF HRV and baroreflex gain independent of respiratory changes. In contrast, we could not demonstrate an increase in HR oscillations during DBT, which is known to activate the baroreflex (Davies et al. 2002). This suggests that the marginal increase in resting HRV was explained from a non-specific central effect rather than increased baroreceptor gain. Furthermore, the increase in LF at rest could be influenced by a slower breathing during the relaxation phase after 10 weeks intervention. However, great care was taken informing participants to breathe normally during rest conditions, and there was no significant change in respiration rate during rest over the 10 -week period. In patients with depression, long-term increases in HRV were found in comparison with healthy controls (Siepmann et al. 2008). Improved resting HRV was observed after respiratory sinus arrhythmia (RSA) biofeedback in persons with PTSD (Zucker et al. 2009). Del Pozo et al. (2004) investigated the effects of HRV BF on patients with coronary artery disease (CAD). HRV was found to increase in the treatment group only. In contrast, others did not find long-term effects on resting HRV (Lehrer et al. 2003; Swanson et al. 2009).

Stress reactivity and recovery

The strength of this study is the extensive autonomic testing which was separated from the treatment sessions. In the present study, treatment effects were seen in HR and HRV in terms of enhanced reactivity to CPT and HGT in the intervention group. Although this could suggest favourable effects of HRV BF on ANS regulation, the results must be interpreted with caution considering the marginal sample size. This is the first study to explore the effects of 
HRV Biofeedback in Neck Pain

HRV BF on recovery from stress in addition to resting periods. The hypothesis of increased parasympathetic activity during recovery from stress was only partly supported in terms of enhanced SDNN after HGT, whereas the other measures of HRV did not show such effects.

A possible explanation concerning the small treatment effects in HRV could be that some individuals in the study group may not have had a severe reduction in HRV in the first place. Hence, the effectiveness of treatment in increasing HRV might have been smaller. In a recent study (Siepmann et al. 2008), no significant increase in HRV was seen for a healthy control group that underwent HRV BF, even though a moderate HRV increase was seen in patients with major depression who received the same treatment.

Subjective ratings

The subjects with chronic neck-shoulder pain who received HRV BF increased their perceived health after 10 weeks, in comparison with controls. More precisely, this improvement was seen for the SF-36 in relation to bodily pain, social function and vitality. In accordance, clinically relevant decreases in pain were found in subjects with fibromyalgia after 10 weekly sessions of HRV BF (Hasset et al. 2007). Overall, HRV BF has been effective in improving various health outcomes (Wheat and Larking 2010).

In the present study, it was hypothesized that self-rated pain would decrease during HRV biofeedback training. However, this was only partly supported by an improvement in bodily pain assessed with SF-36, whereas no treatment effect was revealed from pain assessed with Borg CR-10 scale. It is well known that HRV BF acutely affects ANS regulation (Hassett et al. 2007; Wheat and Larking 2010). It was, thus, expected that improvement in symp- 
HRV Biofeedback in Neck Pain

toms would be related to an increase in HRV. However, as only marginal changes were observed in HRV, this was not sufficiently supported. In contrast, Nolan et al. (2005) demonstrated an association between augmented vagal activation and reduced stress perception after five sessions HRV biofeedback in patients with coronary heart disease. Similarly, a controlled pilot study on subjects with PTSD showed that a change in HRV during HRV BF treatment was able to predict PTSD symptom reduction (Zucker et al. 2009).

In the present study, many of the subjective reports showed improvement over time for both groups, which indicate that the attention paid to the control group could have influenced the results. Even though the treatment group showed reduction in pain and disability ratings after 10 weeks, the interactions were not significant, perhaps due to the high variability and small sample size. Furthermore, the present patient group showed relatively low pain intensity at the moment of intervention, , and mild disability compared to other studies on chronic neck pain (Gockel et al. 1995; Mcarthy et al. 2007). This may have created floor effects. As the present study included participants within a wide range of pain, it is possible that this variability also could have influenced the results.

Implications for future research

Future studies may benefit from including persons with more advanced symptoms. In addition to physical stressors such as HGT and CPT it is important to test autonomic responses to mental stressors as well. It would also be interesting to compare HRV BF with another form of non-invasive treatment known to affect ANS regulation, such as prescribed physical activity. 
HRV Biofeedback in Neck Pain

Conclusion

The present pilot study showed improvement in perceived health over 10 weeks intervention with HRV-biofeedback in subjects with stress-related chronic neck-shoulder pain. Increased resting HRV as well as enhanced reactivity to HGT and CPT might reflect beneficial effects on ANS regulation, and may further suggest that this intervention protocol is suitable for a larger controlled trial. 
HRV Biofeedback in Neck Pain

\section{References}

Berntson, G. G., Bigger, T. Jr., Eckberg, D. L., Grossman, P., Kaufmann, P. G., Malik, M., Nagaraja, H. N., Porges, S.W., Saul, P., Stone, P.H, \& Van Der Molen, M.W. (1997). Heart rate variability: Origin, methods, and interpretive caveats. Psychophysiology, 34, 623-648.

Bongers, P.M., Kremer, A.M., \& ter Laak, J. (2002). Are psychosocial factors, risk factors for symptoms and signs of the shoulder, elbow, or hand/wrist?: a review of the epidemiological literature. American journal of industrial medicine, 41, 315-342.

Borg, G. (1998). Borg's perceived exertion and pain scales. Champaign, IL: Human Kinetics.

Clauw, D.J., Williams, D.A. (2002). Relationship between stress and pain in work-related upper extremity disorders: the hidden role of chronic multi symptom illnesses. American journal of industrial medicine, 41, 370-382.

Davies, L.C., Colhoun, H., Coats, A.J.S., Piepoli, M. \& Francis, D.P. (2002). A noninvasive measure of baroreflex sensitivity without blood pressure measurement. American Heart Journal, 143, 441-447.

Del Pozo, J., Gevirtz, R., Scher, B., \& Guarneri, E. (2004). Biofeedback treatment increases heart rate variability in patients with known coronary artery disease. American Heart Journal, 147, G1-G6.

Fagius, J., Karhuvaara, S., \& Sundlof, G. (1989). The cold pressor test: effects on sympathetic nerve activity in human muscle and skin nerve fascicles. Acta Physiologica Scandinavia, 137(3), 325-334.

Gevirtz, R. (2006) The muscle spindle trigger point model of chronic pain. Biofeedback, 34, 5356.

Gockel, M., Lindholm, H., Alaranta, H., Viljanen, A., Lindquist, A., \& Lindholm, T. (1995). Cardiovascular functional disorder and stress among patients having neck-shoulder symptoms. Annals of the Rheumatic Diseases, 54(6), 494-497.

Hassett, A. L., Radvanski, D.C., Vaschillo, E.G., Vaschillo, B., Sigal, L.H. et al. (2007). A pilot study of the efficacy of heart rate variability (HRV) biofeedback in patients with fibromyalgia. Applied Psychophysiology and Biofeedback, 32(1), 1-10.

Hubbard, D. \& Berkhoff, G. (1993). Myofascial trigger points show spontaneous needle EMG activity. Spine, 18, 1803-1807.

Johansson, H., Windhorst, U., Djupsjöbacka, M., Passatore, M. (eds) (2003). Chronic workrelated myalgia. Neuromuscular mechanisms behind work-related chronic muscle pain syndromes. Gävle University press.

Kalezic, N., Åsell, M., Kerschbaumer, H., \& Lyskov, E. (2007). Physiological Reactivity to Functional Tests in Patients with Chronic Low Back Pain. Journal of Musculoskeletal Pain, 15(1), 29 - 40.

Kalezic, N., Noborisaka, Y., Nakata, M., Crenshaw, A., Karlsson, S., Lyskov, E., \& Eriksson, P-O. (2010). Cardiovascular and muscle activity during chewing in whiplash-associated disorders (WAD). Archives of Oral Biology 55, 447-453.

Kapitza, K.P., Passie, T., Bernateck, M., \& Karst, M. (2010). First non-contingent respiratory biofeedback placebo versus contingent biofeedback in patients with chronic low back pain: a randomized controlled, double-blind trial. Applied Psychophysiology \& Biofeedback, 35, 207-217. 
Khurana, R.K., \& Setty, A. (1996). The value of isometric hand-grip test - studies in various autonomic disorders. Clinical Autonomic Research, 6, 211-218.

Larsson, S. E., Larsson, R., Zhang, Q., Cai, H., \& Öberg, P.Å. (1995). Effects of psychophysiological stress on trapezius muscles blood flow and electromyography during static load. European Journal of Applied Physiology and Occupational Physiology, 71, 493-498.

Larsman, P., Thorn, S., Søgaard, K., Sandsjö, L., Sjøgaard, G., \& Kadefors, R. (2009). Work related perceived stress and muscle activity during standardized computer work among female computer users. Work: A Journal of Prevention, Assessment and Rehabilitation 32(2), 189-199.

Lehrer, P. M., Vaschillo, E., Vaschillo, B., Lu, S. E., Eckberg, D. L., Edelberg, R., et al. (2003). Heart rate variability biofeedback increases baroreflex gain and peak expiratory flow. Psychosomatic Medicine, 65(5), 796-805.

Lehrer, P. M., Vaschillo, E., \& Vaschillo, B. (2000). Resonant frequency biofeedback training to increase cardiac variability: rationale and manual for training. Applied Psychophysiology and Biofeedback, 25(3), 177-91.

Leistad, R., Nilsen, K., Stovner, L., Westgaard, R., Rø,M., Sand, T. (2008) Similarities in stress physiology among patients with chronic pain and headache disorders: evidence for a common pathophysiological mechanism? The Journal of Headache and Pain, 9, 165-175

Lovallo, W. (1975). The cold pressor test and autonomic function. A review and integration. Psychophysiology 12(3), 268-282.

Linton, S.J. (2000). A review of psychological risk factors in back and neck pain. Spine 25, $1148-1156$.

Lisspers, J., Nygren, A., \& Soderman, E. (1997). Hospital Anxiety and Depression Scale (HAD): some psychometric data for a Swedish sample. Acta Psychiatrica Scandinavica, 96(4), 281-6.

McCarthy, M.J.H., Grevitt, M.P., Silcocks, P., \& Hobbs, G. (2007). The reliability of the Vernon and Mior neck disability index, and its validity compared with the short form-36 health survey questionnaire. European Spine Journal, 16(12), 2111-2117.

MacDermid, J. C., Walton, D. M., Avery, S., Blanchard, A., Etruw, E., McAlpine, C., et al. (2009). Measurement properties of the neck disability index: a systematic review. $J$ Journal of Orthopaedic \& Sports Physical Therapy, 39(5), 400-417.

Maekawa, K., Clark ,G.T., Kuboki, T. (2002) Intramuscular hypoperfusion, adrenergic receptors, and chronic muscle pain. The Journal of Pain, 3, 251-260.

Nolan, R., Kamath, M., Floras, J., Stanley, J., Pang, C., Picton, P., et al. (2005). Heart rate variability biofeedback as a behavioral neurocardiac intervention to enhance vagal heart rate control. American Heart Journal, 149, 1137.e1-1137.e7.

Passatore, M., \& Roatta, S. (2006). Influence of sympathetic nervous system on sensorimotor function: whiplash associated disorders (WAD) as a model. European Journal of Applied Physiology, 98, 423-449.

Roatta, S., Arendt-Nielsen, L., Farina, D. (2008). Sympathetic-induced changes in discharge rate and spike-triggered average twitch torque of low-threshold motor units in humans. Journal of Physiology, 586, 5561-557.

Siepmann, M., Aykac, V., Unterdörfer, J., Petrowski, K., Mueck-Weymann, M. (2008) A pilot study on the effects of heart rate variability biofeedback in patients with depression and in healthy subjects. Applied Psychophysiology and Biofeedback, 33, 195-201. 
Swanson, K.S., Gevirtz, R.N.,Brown, M., Spira, J., Guarneri, E., \& Stoletniy, L. (2009). The effects of biofeedback on function in patients with heart failure. Applied Psychophysiology and Biofeedback, 34, 71-91.

Statistics Sweden (2008). Arbetsmiljön 2007 (The Work Environment 2007). In: Arbetsmiljöstatistisk Rapport 2008:4. Arbetsmiljöverket, Stockholm, Sweden.

Task Force of the European Society of cardiology and the North American Society of Pacing and Electrophysiology (1996). Heart rate variability: standards of measurement, physiological interpretation, and clinical use. European Heart Journal, 93, 1043-1065

Vaschillo, E.G., Vaschillo, B., Lehrer, P. (2006). Characteristics of resonance in heart rate variability stimulated by biofeedback. Applied Psychophysiology and Biofeedback, 31(2), 129-139.

Vernon, H. \& Mior, S. (1991). The Neck Disability Index: a study of reliability and validity. Journal of Manipulative Physiological Therapeutics, 14, 409-415.

Victor, R. G., Leimbach, W. N., Jr., Seals, D. R., Wallin, B. G., \& Mark, A. L. (1987). Effects of the cold pressor test on muscle sympathetic nerve activity in humans. Hypertension, 9(5), 429-436.

Ware, J. E., Jr., Gandek, B., Kosinski, M., Aaronson, N. K., Apolone, G., Brazier, J., et al. (1998). The equivalence of SF-36 summary health scores estimated using standard and country-specific algorithms in 10 countries: results from the IQOLA Project. International Quality of Life Assessment. Journal of Clinical Epidemiology, 51(11), 1167-1170.

Wheat, A.L. \& Larkin, K.T. (2010) Biofeedback of heart rate variability and related physiology: A critical review. Applied Psychophysiology and Biofeedback, 35, 229-242)

Zigmond, A.S., \& Snaith, R.P. (1983). The hospital anxiety and depression scale. Acta Psychiatrica Scandinavica, 67, 361-370.

Zucker, T., Samuelson, K., Muench, F., Greenberg, M., Gevirtz, R. (2009). The Effects of Respiratory Sinus Arrhythmia Biofeedback on Heart Rate Variability and Posttraumatic Stress Disorder Symptoms: A Pilot Study. Applied Psychophysiology and Biofeedback, 34, 135143. 
HRV Biofeedback in Neck Pain

Table 1 T-tests and Chi square for equality of patient characteristics before the intervention, and means and standard deviations (sd) for treatment and control groups, respectively.

\begin{tabular}{lcccc}
\hline & Treatment $(n=12)$ & Controls $(n=10)$ & & \\
Characteristics (scores) & $n /$ Mean $(s d)$ & $n /$ Mean $(s d)$ & $t / X^{2}$ & $p$ \\
\hline Women & 11 & 10 & 0.18 & 0.89 \\
BMI $\left(\mathrm{kg} / \mathrm{m}^{2}\right)$ & 23.8 & 24.1 & 0.21 & 0.83 \\
Age & 39.6 & 42.9 & 1.10 & 0.28 \\
On sick leave & 2 & 0 & 1.83 & 0.18 \\
Pain duration (years) & $5.7(5.5)$ & $6.0(3.4)$ & -0.13 & 0.90 \\
Average pain intensity - Borg (0-10) & $3.4(0.8)$ & $3.4(1.2)$ & 0.15 & 0.88 \\
Current pain intensity - Borg (0-10) & $2.6(1.3)$ & $2.5(1.1)$ & 0.16 & 0.88 \\
Symptoms - SMSS (0-98) & $33.2(9.4)$ & $36.8(15.1)$ & 0.70 & .49 \\
\hline
\end{tabular}


HRV Biofeedback in Neck Pain

Table 2 Repeated measure ANOVA, with $p$-values, $F$-values and effect size $\left(n^{2}\right)$, and means (sd) for subjective reports, pre- and post- intervention

\begin{tabular}{|c|c|c|c|c|c|c|c|}
\hline \multirow[t]{2}{*}{ Symptoms (specific Scores) } & \multicolumn{2}{|c|}{$\begin{array}{l}\text { Treatment group } \\
\qquad \text { M (sd) }\end{array}$} & \multicolumn{2}{|c|}{$\begin{array}{l}\text { Control group } \\
\qquad \mathrm{M}(\mathrm{sd})\end{array}$} & \multicolumn{3}{|c|}{$\begin{array}{c}\text { ANOVA } \\
(\text { groupXtime) }\end{array}$} \\
\hline & Before & After & Before & After & $F$ & $p$ & $n^{2}$ \\
\hline Current Pain (Borg) (0-10) & $2.6(1.3)$ & $1.7(1.4)$ & $2.5(1.1)$ & $2.0(1.7)$ & 0.72 & $.41^{*}$ & 0.03 \\
\hline NDI (0-100) & $21.3(7.5)$ & $14(10)$ & $25.6(15.2)$ & $20.6(14.4)$ & 0.27 & $.61 *$ & 0.01 \\
\hline \multicolumn{8}{|l|}{ SF-36 } \\
\hline Physical function & $89.6(7.2)$ & $92.5(7.8)$ & $77.5(17.0)$ & $84.5(15.2)$ & 1.27 & $.27 *$ & 0.06 \\
\hline Role physical & $60.4(43)$ & $77.1(42)$ & $57.5(37.5)$ & $67.5(39.2)$ & 0.20 & .67 & 0.01 \\
\hline Bodily pain & $46.5(21)$ & $71.8(18)$ & $49.9(18.2)$ & $58.4(39.2)$ & 4.39 & $.049 *$ & 0.18 \\
\hline General health & $60.8(22)$ & $63.4(24)$ & $61.4(23.2)$ & $60.5(25.26)$ & 0.21 & .65 & 0.01 \\
\hline Vitality & $37.1(22)$ & $57.5(22)$ & $49.0(27.4)$ & $48.0(29.7)$ & 9.69 & $.005^{*}$ & 0.32 \\
\hline Social function & $76.0(23)$ & $90.612)$ & $85.0(24.2)$ & $82.5(24.4)$ & 4.50 & .047 & 0.18 \\
\hline Role emotional & $72.2(40)$ & $83.3(33)$ & $86.7(28.1)$ & $83.3(28.3)$ & 3.68 & .069 & 0.16 \\
\hline Mental health & $66.3(20)$ & $72.1(18)$ & $69.9(18.2)$ & 72.8 (21.7) & 0.28 & .60 & 0.01 \\
\hline \multicolumn{8}{|l|}{ HAD (0-20) } \\
\hline Anxiety & $6.83(2.52)$ & $5.50(3.06)$ & $7.64(4.15)$ & $6.45(3.59)$ & 0.02 & $.87 *$ & 0.01 \\
\hline Depression & $3.50(3.37)$ & $2.42(2.71)$ & $6.27(5.18)$ & $4.91(4.46)$ & 0.08 & $.78^{*}$ & 0.01 \\
\hline SMSS (0-98) & $33.2(9.44)$ & $26.8(16.0)$ & $36.8(15.05)$ & $35.6(18.0)$ & 1.09 & .31 & \\
\hline
\end{tabular}

Note: *Significant time effect $(p<0.05)$; Significant $(p<0.05)$ interaction effects (group X time) are bold faced 
HRV Biofeedback in Neck Pain

Table 3 Resting HRV with means (sd) for treatment (T) and control (C) groups before and after treatment. ANOVA show $F$-values, $p$-values and effect size $\left(n^{2}\right)$ for interaction effects

\begin{tabular}{|c|c|c|c|c|c|c|}
\hline \multirow[t]{2}{*}{ Measure } & \multirow[b]{2}{*}{ Group } & \multirow{2}{*}{$\frac{\text { Before }}{\mathrm{M}(\mathrm{sd})}$} & \multirow{2}{*}{$\frac{\text { After }}{\mathrm{M}(\mathrm{sd})}$} & \multicolumn{3}{|c|}{$\begin{array}{c}\text { ANOVA } \\
\text { (groupXtime) }\end{array}$} \\
\hline & & & & $F$ & $p$ & $n^{2}$ \\
\hline \multirow[t]{2}{*}{ IBI (s) } & $\mathrm{T}$ & $0.90(0.11)$ & $0.93(0.11)$ & 3.60 & 0.072 & 0.15 \\
\hline & $\mathrm{C}$ & $0.94(0.09)$ & $0.90(0.09)$ & & & \\
\hline \multirow[t]{2}{*}{ SDNN (s) } & $\mathrm{T}$ & $0.05(0.03)$ & $0.06(0.03)$ & 3.86 & 0.063 & 0.16 \\
\hline & $\mathrm{C}$ & $0.05(0.01)$ & $0.04(0.02)$ & & & \\
\hline \multirow[t]{2}{*}{ pNN50 } & $\mathrm{T}$ & 17 (17) & $22(12)$ & 3.83 & 0.064 & 0.16 \\
\hline & $\mathrm{C}$ & $16(12)$ & $12(12)$ & & & \\
\hline \multirow[t]{2}{*}{$\mathrm{LF}(\log )$} & $\mathrm{T}$ & $5.98(1.4)$ & $6.52(1.5)$ & 6.99 & 0.016 & 0.26 \\
\hline & $\mathrm{C}$ & $6.54(0.8)$ & $6.03(0.8)$ & & & \\
\hline \multirow[t]{2}{*}{ HF $(\log )$} & $\mathrm{T}$ & $6.46(1.7)$ & $6.80(1.1)$ & 1.03 & 0.32 & 0.05 \\
\hline & $\mathrm{C}$ & $6.43(0.9)$ & $6.19(1.0)$ & & & \\
\hline \multirow[t]{2}{*}{$\mathrm{LF} / \mathrm{HF}$ ratio } & $\mathrm{T}$ & $0.84(0.7)$ & $2.56(4.1)$ & 3.11 & 0.093 & 0.13 \\
\hline & $\mathrm{C}$ & $1.76(2.2)$ & $1.06(0.70)$ & & & \\
\hline
\end{tabular}

Note: Significant $(p \leq 0.05)$ interaction effects (group X time) are bold faced.

Table 4 HRV for reactivity to hand grip and cold pressor tests.

ANOVA with $F$-values, $p$-values and effect size $\left(n^{2}\right)$ for interaction effects (group X time)

\begin{tabular}{lccccccc}
\hline \multirow{3}{*}{ Measure } & \multicolumn{3}{c}{ Hand grip } & & \multicolumn{3}{c}{ Cold pressor } \\
& \multicolumn{2}{c}{ ANOVA (group X time) } & & \multicolumn{2}{c}{ ANOVA (group X time) } \\
\cline { 2 - 4 } \cline { 7 - 8 } & $F$ & $p$ & $n^{2}$ & & $F$ & $p$ & $n^{2}$ \\
\hline IBI (s) & 7.84 & $\mathbf{0 . 0 1 1}$ & 0.28 & & 6.57 & $\mathbf{0 . 0 2}$ & 0.27 \\
SDNN (s) & 4.03 & 0.058 & 0.17 & & 0.80 & 0.38 & 0.04 \\
pNN50 & 4.35 & $\mathbf{0 . 0 5 0}$ & 0.18 & & 10.34 & $\mathbf{0 . 0 0 5}$ & 0.37 \\
LF (log) & 6.1 & $\mathbf{0 . 0 2 3}$ & 0.25 & & 0.62 & 0.45 & 0.05 \\
HF (log) & 1.83 & 0.19 & 0.09 & & 3.01 & 0.11 & 0.19 \\
LF/HF ratio & 4.12 & 0.057 & 0.19 & & 0.04 & 0.84 & 0.01
\end{tabular}

Note: Significant $(p \leq 0.05)$ interaction effects (group X time) are bold faced. 
Table 5 Means (M) and standard deviations (sd) of HRV measures under rest and reactivity and recovery to HGT and CPT for treatment (T) and control (C) groups, before and after treatment (Table 5 might be placed in the appendix or excluded).

\begin{tabular}{|c|c|c|c|c|c|c|c|c|c|c|c|}
\hline \multirow[t]{4}{*}{ Measure } & & \multirow{2}{*}{\multicolumn{2}{|c|}{$\begin{array}{c}\text { Baseline } \\
\text { Rest }\end{array}$}} & \multicolumn{4}{|c|}{ Hand grip } & \multicolumn{4}{|c|}{ Cold pressor } \\
\hline & \multirow[b]{3}{*}{ Group } & & & \multirow{2}{*}{\multicolumn{2}{|c|}{$\begin{array}{l}\text { Reactivity } \\
\text { M (sd) }\end{array}$}} & \multirow{2}{*}{\multicolumn{2}{|c|}{$\begin{array}{c}\text { Recovery } \\
\text { M (sd) }\end{array}$}} & \multirow{2}{*}{\multicolumn{2}{|c|}{$\begin{array}{c}\text { Reactivity } \\
\text { M (sd) }\end{array}$}} & \multirow{2}{*}{\multicolumn{2}{|c|}{$\begin{array}{c}\text { Recovery } \\
\text { M (sd) }\end{array}$}} \\
\hline & & \multicolumn{2}{|c|}{$\mathrm{M}(\mathrm{sd})$} & & & & & & & & \\
\hline & & Before & After & Before & After & Before & After & Before & After & Before & After \\
\hline \multirow[t]{4}{*}{ IBI (s) } & \multirow[t]{2}{*}{$\mathrm{T}$} & 0.90 & 0.93 & -0.19 & $-0.21 *$ & 0.18 & 0.21 & -0.08 & $-0.11 *$ & 0.08 & 0.11 \\
\hline & & $(0.11)$ & $(0.11)$ & (0.09) & $(0.13)$ & $(0.10)$ & $(0.13)$ & $(0.08)$ & $(0.09)$ & $(0.08)$ & $(0.10)$ \\
\hline & \multirow[t]{2}{*}{$\mathrm{C}$} & 0.94 & 0.90 & -0.18 & -0.13 & 0.13 & 0.12 & -0.14 & -0.09 & 0.12 & 0.11 \\
\hline & & (0.09) & (0.09) & $(0.08)$ & $(0.01)$ & $(0.04)$ & (0.09) & $(0.11)$ & $(0.11)$ & $(0.11)$ & $(0.13)$ \\
\hline \multirow[t]{4}{*}{ SDNN } & \multirow[t]{2}{*}{$\mathrm{T}$} & 0.05 & 0.06 & -0.02 & -0.03 & 0.03 & $0.05 *$ & 0.01 & 0.00 & 0.00 & 0.01 \\
\hline & & $(0.03)$ & $(0.03)$ & $(0.03)$ & $(0.01)$ & $(0.04)$ & $(0.04)$ & $(0.01)$ & $(0.03)$ & $(0.02)$ & $(0.03)$ \\
\hline & \multirow[t]{2}{*}{$\mathrm{C}$} & 0.05 & 0.04 & -0.01 & -0.01 & 0.02 & 0.02 & 0.00 & 0.01 & 0.01 & 0.01 \\
\hline & & $(0.01)$ & (0.02) & $(0.01)$ & $(0.01)$ & $(0.03)$ & $(0.01)$ & $(0.01)$ & $(0.02)$ & $(0.02)$ & $(0.02)$ \\
\hline \multirow[t]{4}{*}{ pNN50 } & \multirow[t]{2}{*}{$\mathrm{T}$} & 0.28 & 0.35 & -0.26 & -0.33 & 0.27 & 0.36 & -0.03 & -0.15 & -0.02 & 0.15 \\
\hline & & $(0.27)$ & $(0.21)$ & $(0.26)$ & $(0.21)$ & $(0.26)$ & $(0.20)$ & $(0.13)$ & $(0.13)$ & $(0.13)$ & $(0.17)$ \\
\hline & \multirow[t]{2}{*}{$\mathrm{C}$} & 0.27 & 0.19 & -0.22 & -0.13 & 0.16 & 0.17 & -0.16 & -0.07 & 0.15 & 0.15 \\
\hline & & $(0.20)$ & $(0.19)$ & $(0.19)$ & $(0.15)$ & $(0.17)$ & $(0.18)$ & $(0.15$ & $(0.12$ & $(0.12)$ & $(0.16)$ \\
\hline \multirow{4}{*}{$\begin{array}{l}\text { LF } \\
(\log )\end{array}$} & \multirow[t]{2}{*}{$\mathrm{T}$} & 5.98 & $6.52 *$ & -0.11 & $-1.14^{*}$ & 0.41 & 1.33 & 0.50 & 0.20 & -0.32 & -0.13 \\
\hline & & (1.4) & $(1.5)$ & (1.1) & $(.9)$ & (1.43) & (1.13) & $(0.93)$ & (1.87) & (1.41) & (1.57) \\
\hline & \multirow[t]{2}{*}{$\mathrm{C}$} & 6.54 & 6.03 & -0.68 & -0.9 & 0.46 & 0.77 & -0.26 & 0.05 & 0.13 & 0.55 \\
\hline & & $(0.8)$ & $(0.8)$ & (1.1) & (1.5) & (1.12) & $(0.92)$ & $(1.08)$ & $(0.97)$ & $(1.19)$ & $(1.17)$ \\
\hline \multirow{4}{*}{$\begin{array}{l}\mathrm{HF} \\
(\log )\end{array}$} & \multirow[t]{2}{*}{$\mathrm{T}$} & 6.46 & 6.80 & -1.49 & -1.65 & 1.39 & 1.83 & -0.07 & -0.44 & -0.03 & 0.15 \\
\hline & & $(1.7)$ & (1.1) & $(1.1)$ & $(1.4)$ & (1.19) & (1.44) & $(0.56)$ & $(0.44)$ & $(0.46)$ & $(0.73)$ \\
\hline & \multirow[t]{2}{*}{$\mathrm{C}$} & 6.43 & 6.19 & -1.19 & -0.69 & 0.96 & 0.92 & -0.93 & -0.49 & 0.86 & 0.55 \\
\hline & & $(0.9)$ & $(1.0)$ & $(0.8)$ & $(1.0)$ & $(0.92)$ & $(0.97)$ & (1.21) & $(0.94)$ & $(1.20)$ & $(0.97)$ \\
\hline \multirow{4}{*}{$\begin{array}{l}\mathrm{LF} / \mathrm{HF} \\
\text { ratio }\end{array}$} & \multirow[t]{2}{*}{$\mathrm{T}$} & 0.84 & 2.56 & 3.22 & -0.30 & -2.46 & -0.01 & 0.62 & -0.56 & 0.70 & 0.78 \\
\hline & & $(0.7)$ & (4.1) & $(4.89)$ & $(2.54)$ & $(5.63)$ & $(1.74)$ & $(1.54)$ & $(4.60)$ & $(4.22)$ & $(3.17)$ \\
\hline & \multirow[t]{2}{*}{$\mathrm{C}$} & 1.76 & 1.06 & 0.39 & 3.23 & 0.49 & -1.96 & 2.46 & 0.88 & -2.36 & 0.72 \\
\hline & & $(2.2)$ & $(0.70)$ & (2.67) & (6.61) & (4.43) & $(4.20)$ & (3.30) & $(1.55)$ & (3.13) & (1.63) \\
\hline
\end{tabular}

The HRV reactivity and recovery scores (means and standard deviations) were calculated as the difference between baseline and test and from test to resting condition, respectively, for normal-to-normal R-R intervals (IBI), the standard deviation of normal-to-normal R-R intervals (SDNN), the proportion of successive differences between adjacent R-R intervals >50 milliseconds (pNN50) and for log transformed low frequency power $(\log$ LF), $\log$ transformed high frequency power $(\log \mathrm{HF})$ and the ratio between $\mathrm{LF}$ and $\mathrm{HF}$ power $(\mathrm{LF} / \mathrm{HF})$. *significant group $\mathrm{X}$ time interaction $(p \leq 0.05)$ 
HRV Biofeedback in Neck Pain

\section{Captions for figures}

Figure 1 shows SDNN (ms) during baseline, HGT and rest, pre- and post-treatment for treatment and control groups, respectively 
HRV Biofeedback in Neck Pain

Figure 1

\section{SDNN}

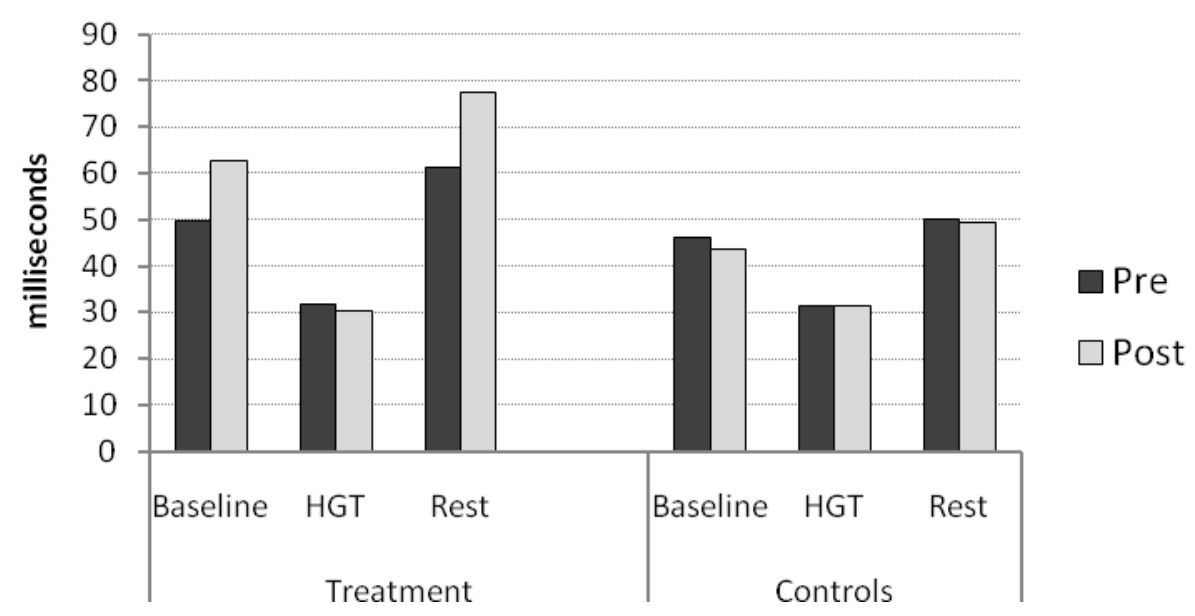

